\title{
The Application of FFT Analysis on the Feeder Current Based on the Smooth Function in MATLAB
}

\author{
Chang Yujie, Li Huawei, Huang Songwei, He Jinghan, Yang Shaobing, Wang Qiang \\ School of Electrical Engineering, \\ Beijing Jiaotong University \\ Beijing 100044, China \\ e-mail: 12291026@bjtu.edu.cn
}

\begin{abstract}
Fast Fourier transform(FFT) is an important tool for harmonic analysis in power systems and a method of FFT analysis of the signal is very important for power systems. For finding and forming a basic way to FFT analysis of feeder current, the paper embarks from the principle of Fourier transform and analyzes the principle and algorithm of the related metrics such as spectrum and signal-to-noise ratio(SNR). Then it uses the smooth function which is based on the moving average filter to filter the feeder current, which is used to prove the feasibility of this method. Meanwhile its result which we can get the data from figures and tables in the paper also shows that the smooth function as a low-pass filter can effectively filter out noise and keep the feeder current fundamental wave using the way that changes span values and method, but it will cause the base wave distortion.
\end{abstract}

Keywords-MATLAB;FFT analysis;spectrum;SNR;feeder current

\section{INTRODUCTION}

In recent years, the rapid development of electrification railway and high-power locomotives run a lot of impact on power system, which is attracting more and more attention. The harmonic that electric locomotive's load produces will cause the decline of power quality, make electrical equipment for heating and threat to the safe operation of the power system[1]. At the same time, the feeder current frequency has a very important role to the related detection of the power system[2]. So the analysis and processing of the harmonic current signal is very important. It needs the basic method to the analysis of the signal to provide the basis for further study.

This paper mainly describes the spectrum analysis and the SNR of estimation based on FFT algorithm, and uses MATLAB to simulation of the feeder current and smooth function analysis. It is also proposed the basic method using FFT to analyze feeder current and the filter of the smooth function.

\section{FSAT FOURIER TRANSFORM(FFT) PRINCIPLE}

We suppose a random signal $\mathrm{X}(\mathrm{t})$. Then we intercept a sample signal from the random signal, which length is $2 \mathrm{~T}$, and recorded as $x_{T}(t)$.Under this assumption, the follow fourier transform is used[3]:

$$
\begin{aligned}
& X_{T}(\omega)=\int_{-\infty}^{+\infty} x_{T}(\mathrm{t}) e^{-j \omega t} d t=\int_{-T}^{T} x_{T}(t) e^{-j \omega t} d t \\
& x_{T}(\mathrm{t})=\frac{1}{2 \pi} \int_{-\infty}^{+\infty} X_{T}(\omega) e^{j \omega t} d \omega
\end{aligned}
$$

Discrete Fourier transform (DFT) is a method to the Fourier transform or frequency analysis of limited length digital signal, and is the Fourier transform in the form of discrete in time domain and frequency domain. We make the periodic extended for limited length of digital signal, that is, we extend periodically digital signal into the long periodic signal. Then it is calculated by using Fourier series, and the discrete Fourier transform (DFT) can be derived:

$$
\begin{aligned}
& \mathrm{X}(\mathrm{k})=\sum_{n=0}^{N-1} x(n) W_{N}^{\mathrm{kn}} \\
& \mathrm{x}(\mathrm{n})=\frac{1}{N} \sum_{k=0}^{N-1} X(k) W_{N}^{-k n}, k=0,1, \ldots, N-1 \\
& \text { Where } W_{n}=\mathrm{e}^{-j \frac{2 \pi}{N}} \text { is called twiddle factor. In (3) and }
\end{aligned}
$$
(4), the periodic and symmetry of coefficient $W_{N}^{\mathrm{kn}}$ are as follows:

$$
\begin{aligned}
& W_{N}^{k n}=W_{N}^{k(n+N)}=W_{N}^{(k+N) n} \\
& W_{N}^{k n+\frac{N}{2}}=-W_{N}^{k n}
\end{aligned}
$$

FFT is a fast algorithm of DFT. By using (5) and (6), a long sequence of DFT can decompose into a short sequence of DFT. In this way, the combination operation of some of items can reduce about half of the computation[4].

\section{RELATED METRICS ANALYSIS OF FFT}

The equations are an exception to the prescribed specifications of this template. You will need to determine whether or not your equation should be typed using either the Times New Roma

We suppose a sine function which is: $y=10 \sin (40 \pi t+\pi / 4)$ 
In MATLAB, we use randn function and (7) to get a noisy signal as the test signal. Its time-domain figure is as follows:

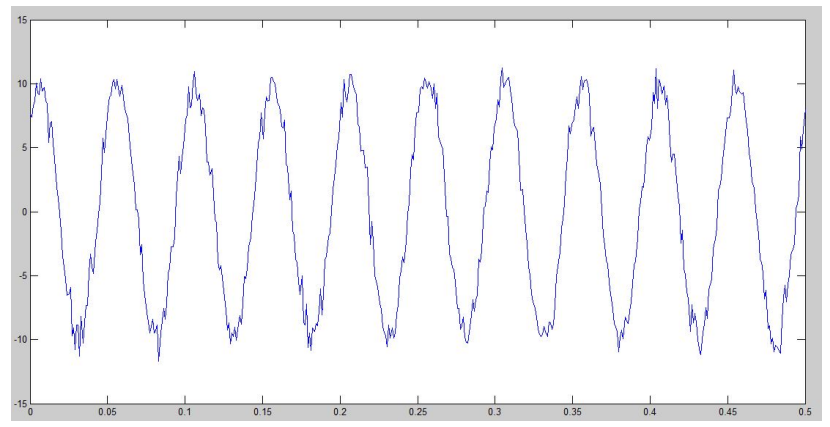

Figure 1. Time-domain figure of test signal

\section{A. Spectrum}

Fourier transform will transform the signal from time domain to frequency domain, which makes us get the information of spectrum. The spectrum diagram can directly reflect the frequency characteristics of signal, and it is convenient for further analysis of signal.

Because of the algorithm of DFT, when selecting sampling point of FFT, the best selection of signal sequence is a basic cycle[5]. In this way, after the periodic extension, signal sequence will be more close to the original sequence, which is the premise of accurate spectrum analysis.

Similarly, if the time window length of signal is in multiples of a basic cycle, it also can achieve such effect. According to the periodic extension, we can know in two cases the signal sequence is same. So the time window length is $\mathrm{N}$ cycle in Fourier transform of signal, which can make the frequency and amplitude frequency of spectrum is very close to the real value after the transformation to the frequency domain, so as to realize the signal processing and analysis[6].

Fig. 2 is the amplitude-frequency maps of the test signal after FFT. It can be seen that there is the amplitude of 9.9832 in $20 \mathrm{~Hz}$, only 0.0168 difference with the theoretical value. The rest of the frequency points are all noise.

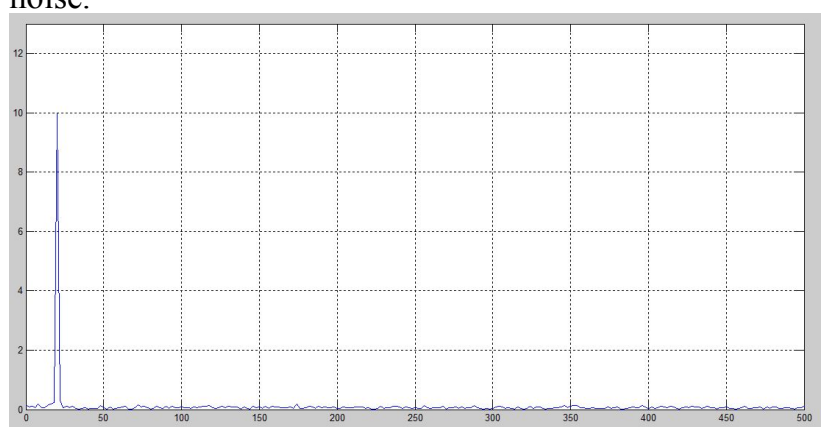

Figure 2. Amplitude-frequency map of test signal.

\section{B. Signal-to noise Ratio(SNR)}

SNR is the ratio for signal average power and noise average power. $X_{T}(\omega)$ and $x_{T}(t)$ in (1) and (2) is as the random function of the experimental results $\mathrm{F}$, so they can be transformed into $X_{T}(\omega, \xi)$ and $x_{T}(\omega, \xi)$. In this case, the signal average power can be written:

$$
W_{\xi}=\frac{1}{2 \pi} \int_{-\infty}^{+\infty} \lim _{T \rightarrow \infty} \frac{1}{2 T}\left|X_{T}(\omega, \xi)\right|^{2} d \omega
$$

We make a statistical average for $\xi$ above, there is

$W=\mathrm{E}\left[W_{\xi}\right]=\frac{1}{2 \pi} \int_{-\infty}^{+\infty} \lim _{T \rightarrow \infty} \frac{1}{2 T} E\left[\left|X_{T}(\omega, \xi)\right|^{2}\right] d \omega$

Where $\mathrm{W}$ is the average power of random signal $\mathrm{X}(\mathrm{t})$.

The signal average power $\mathrm{S}$ and the noise average power $\mathrm{N}$ calculated are taken in (10), the result is the SNR[7].

$$
\mathrm{SNR}=10 \lg \frac{5}{N}(\mathrm{~dB})
$$

If the signal is the digital signal, its FFT transform is (3). According to Parseval's theory, there is

$$
\sum_{n=0}^{N-1}|x(n)|^{2}=\frac{1}{N} \sum_{k=0}^{N-1}|X(k)|^{2}
$$

We can see that energy in the time domain and frequency domain is conserved from (11)[8]. Signal sequence by FFT transformation to the frequency domain, the energy will be converted to the frequency domain. Through the analysis and calculation of the frequency domain energy, we can estimate the SNR.

For the single frequency signal, when the signal frequency is located in the observation frequency point after FFT transform, the energy is concentrated almost entirely in the frequency point. At this time, the remaining frequency points' amplitude is regarded as noise, so we can estimate the SNR. But when the signal frequency is not located in the observation frequency, the quadratic sum of the frequency point which has the maximum amplitude and the surrounding $2 \mathrm{~N}$ frequency points is as the ideal signal, and the remaining frequency points are as noise. The formula for calculating is:

$$
\mathrm{SNR}=10 \lg \left(\frac{\sum_{i=-N}^{N} A_{K+i}^{2}}{\sum_{j=K+\mathrm{i}+1}^{M} A_{j}^{2}}\right)
$$

Where $\mathrm{M}$ is the number of FFT point, $A_{j}$ and $A_{K+\mathrm{i}}$ are the amplitude of signal frequency point[9].

For the test signal, considering amplitude-frequency map, it is the single frequency signal. Taken ten cycle time window length, the frequency of the signal is observed after FFT transform. So the signal power can be expressed as the square of the amplitude which is the maximum, and noise power is for the rest of the frequency amplitude. Taking them in (12), we can get the SNR $=18.7291$

\section{BASIC PRINCIPLE AND USAGE OF SMOOTH FUNCTION}

Moving average filter is a lowpass filter. It makes the continuous sampling data as a queue length on for N. After a new measurement, the first datum is deleted, the rest $\mathrm{N}$ 1 data are in turn forward and the new sampling datum is inserted as the new queue' tail. Then the result of arithmetic operations for the queue is used as a result of the measurement. It is a supplement to the analog filter and used for real-time detection. As long as the sampling rate is high enough, it can get the ideal result. 
Smooth function is a function using the moving average filter to data processing and filtering. It smooths the data in column vector $y[10]$.

Six filter methods of smooth function is given in the table below:

TABLE I. FILTER METHOD OF SMOOTH FUNCTION

\begin{tabular}{|c|c|}
\hline method & Description \\
\hline moving & $\begin{array}{l}\text { Moving average } \text { (default). A } \\
\text { lowpass filter with filter } \\
\text { coefficients equal to the reciprocal } \\
\text { of the span. }\end{array}$ \\
\hline lowess & $\begin{array}{l}\text { Local regression using weighted } \\
\text { linear least squares and a } 1 \text { st degree } \\
\text { polynomial model }\end{array}$ \\
\hline loess & $\begin{array}{l}\text { Local regression using weighted } \\
\text { linear least squares and a 2nd } \\
\text { degree polynomial model }\end{array}$ \\
\hline sgolay & $\begin{array}{l}\text { Savitzky-Golay filter. A } \\
\text { generalized moving average with } \\
\text { filter coefficients determined by an } \\
\text { unweighted linear least-squares } \\
\text { regression and a polynomial model } \\
\text { of specified degree (default is 2). } \\
\text { The method can accept nonuniform } \\
\text { predictor data. }\end{array}$ \\
\hline rlowess & $\begin{array}{l}\text { A robust version of 'lowess' that } \\
\text { assigns lower weight to outliers in } \\
\text { the regression. The method assigns } \\
\text { zero weight to data outside six } \\
\text { mean absolute deviations. }\end{array}$ \\
\hline rloess & $\begin{array}{l}\text { A robust version of 'loess' that } \\
\text { assigns lower weight to outliers in } \\
\text { the regression. The method assigns } \\
\text { zero weight to data outside six } \\
\text { mean absolute deviations. }\end{array}$ \\
\hline
\end{tabular}

\section{FFT ANALYSIS OF FEEDER CURRENT BY MATLAB}

In the paper, we use FFT related indicators to analyze the feeder current under the traction working condition from a certain traction substation.

\section{A. Analysis of Original Signal}

The feeder current signal in the time domain is shown in Fig. (3).After FFT analysis of that intercepted two cycles from the original signal, we can get the signal spectrum shown in Fig. (4). As can be seen from it, the signal includes fundamental wave of $50 \mathrm{~Hz}$ and all the odd harmonics, for example: $150 \mathrm{~Hz}, 250 \mathrm{~Hz}, 350 \mathrm{~Hz}$, etc.

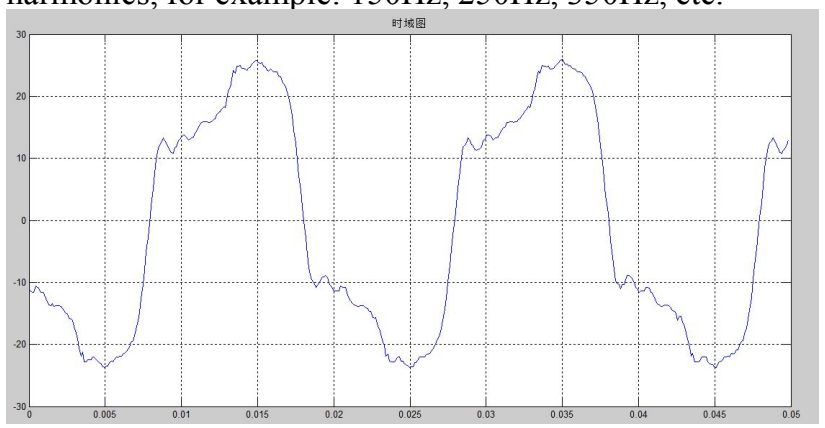

Figure 3. Time-domain figure of feeder current

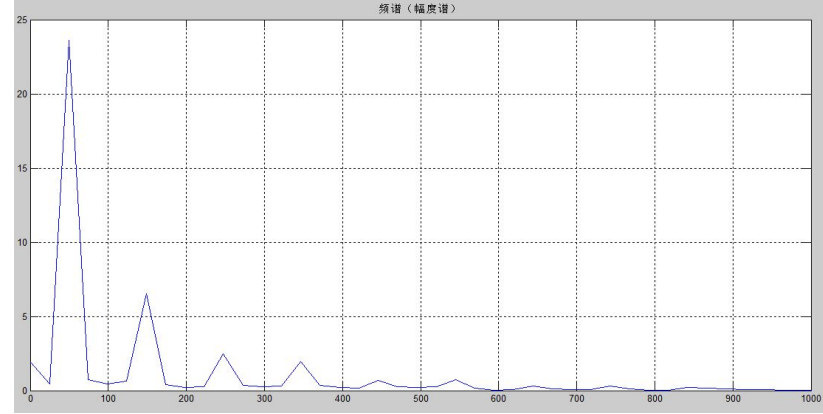

Figure 4. Amplitude-frequency map of feeder current

The exist of every harmonic is the characteristic of the traction power supply when the train runs at low speed. The further study of the signal hopes to filter every harmonic and reserve the fundamental wave of $50 \mathrm{~Hz}$.So amplitude in $50 \mathrm{~Hz}$ can be as the signal power, and the remaining frequency point amplitude is the noise power. Numerical generation will be calculated into (11) to get $\mathrm{SNR}=9.8168$.

\section{B. Analysis of Signal Which is Filtered by Smooth Function}

We use smooth function to process the feeder current collected, whose analysis is as a MATLAB example to analyze the application of FFT.

1) Changing span of the moving average

We change the span value of the smooth function to process the current signal and get the time-domain figure as shown in Fig. 5, the frequency-domain figure as shown in Fig. 6. From these, we can get the SNR of the signal and the amplitude at $50 \mathrm{~Hz}$ in various circumstances as shown in TABLE II.

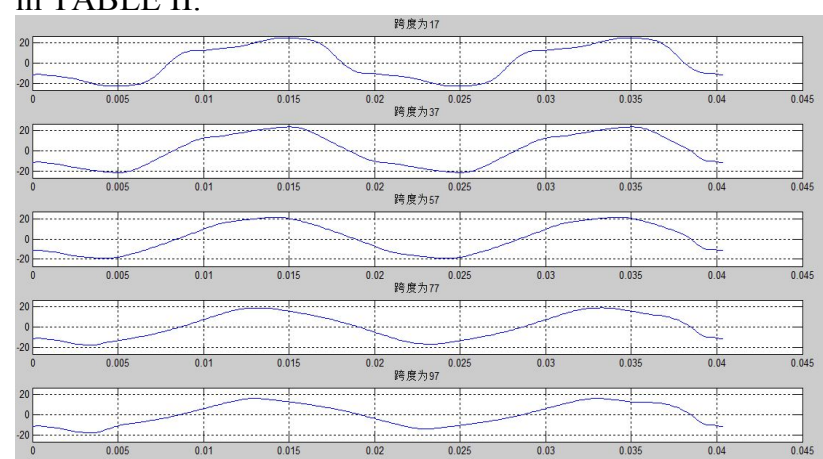

Figure 5. Time-domain figure of signals which are filtered by different span value

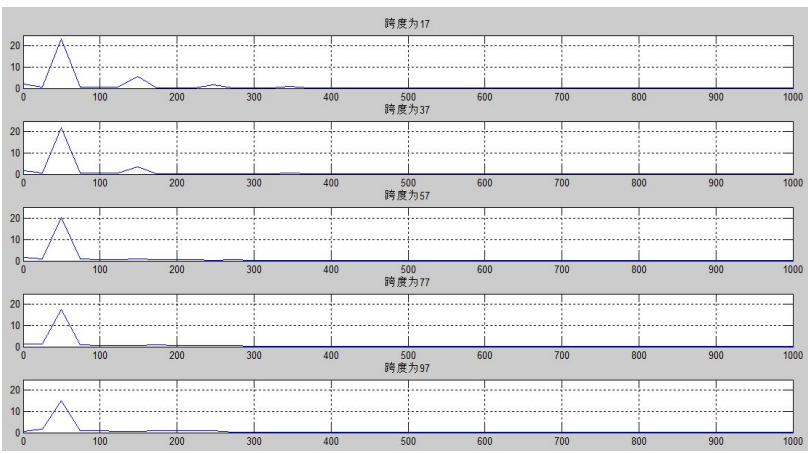

Figure 6. Frequency-domain figure of signals which are filtered by different span value 
TABLE II. SNR AND AMPLITUDE AT 50Hz OF SIGNALS WHICH ARE FILTERED BY DIFFERENT SPAN VALUE

\begin{tabular}{|c|c|c|}
\hline Span value & SNR & Amplitude at 50Hz \\
\hline 17 & 11.3229 & 23.2904 \\
\hline 37 & 15.0483 & 22.0765 \\
\hline 57 & 18.5866 & 20.0802 \\
\hline 77 & 17.1865 & 17.6133 \\
\hline 97 & 14.6285 & 15.1027 \\
\hline
\end{tabular}

Through the analysis of the above figure and table, we can get that smooth function as a low-pass filter has a great effect on the harmonic signal filtering, but greatly influences the fundamental wave, and the signal distortion is more serious. For this feeder current, when the span value is 57 , SNR is the largest, and it has the best filtering effect.

2) Changing method of the smooth function

We can use the same way above to change the method of the smooth function to process the current signal and get Fig. (7) and Fig. (8) and TABLE III

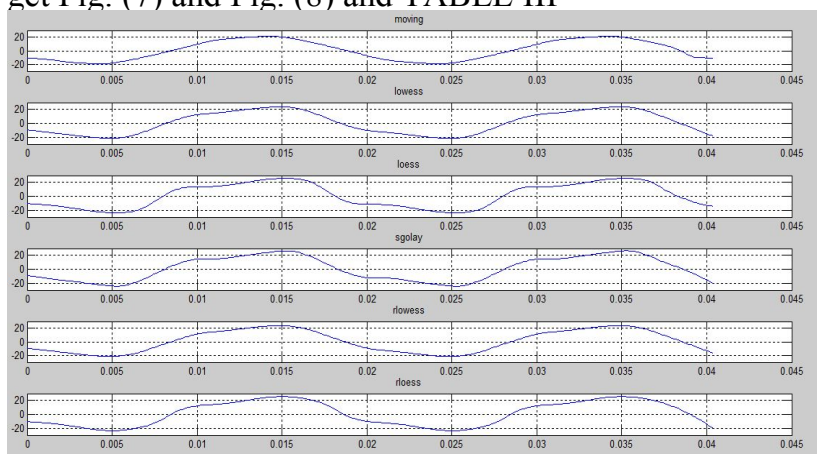

Figure 7. Time-domain figure of signals which are filtered by different method

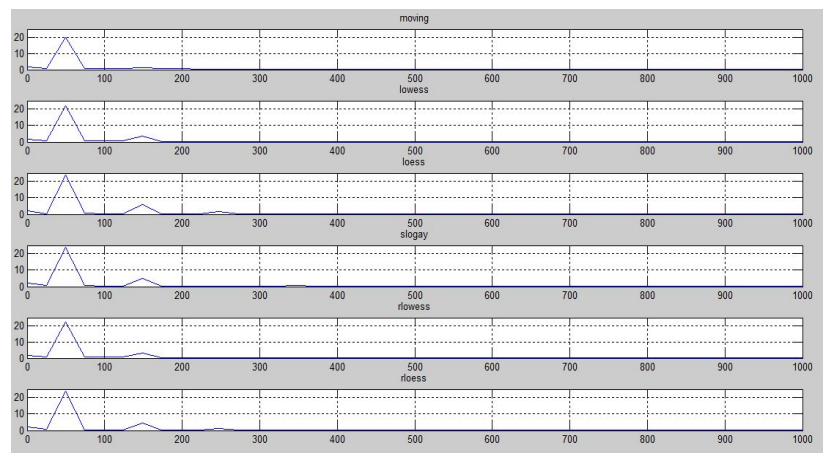

Figure 8. Time-domain figure of signals which are filtered by different method
TABLE III. SNR AND AMPLITUDE AT 50Hz OF SIGNALS WHICH ARE FILTERED BY DIFFERENT METHOD

\begin{tabular}{|c|c|c|}
\hline method & SNR & Amplitude at 50Hz \\
\hline moving & 18.5866 & 20.0802 \\
\hline lowess & 14.5234 & 22.1501 \\
\hline loess & 11.3413 & 23.6486 \\
\hline slogay & 12.4844 & 23.6482 \\
\hline rlowess & 15.8707 & 22.2239 \\
\hline rloess & 13.6345 & 24.0263 \\
\hline
\end{tabular}

As can be seen from these above, the method 'moving' is the best method for this feeder current, at the same time, the signal distortion is the most serious.

\section{CONCLUSION}

In this paper, we analyze the feeder current of traction substation. Spectrum, SNR and other related indicators can analyze the signal itself and the filtering effect more accurately and provide favorable data. By the simulation we can see that the smooth function is the moving average filtering of signal. Though it can effectively filter out noise by selecting the span value and filtering method, it causes the base wave more less than before because of the existence of averaging process. In the case of the combination of the relevant theories and examples analysis, we can improve the students' analysis ability and practice ability.

\section{REFERENCES}

[1] M. H. Jiang, X. J. Wang and J. Lou, "Analysis and Prediction of Harmonic Current in Feeder of Traction Substation," Power System Technology, vol. 38(1), 2014, pp. 3829-3833.

[2] B. Lang and M. L. Wu, "Harmonics Model of Traction Network and Its Simulation," Automation of Electric Power Systems, vol. 33, Sept.2009, pp. 76-80

[3] W. K. Edward and S. H. Bonnie, Fundamentals of Signals and Systems Using the Web and MATLAB. Beijing: Electronic Industry, 2007.

[4] Z. L. Wang and M. Liu, Proficient in MATLAB. Beijing: Electronic Industry, 2006.

[5] H. W. Li and Z. Y. Li, "FFT Alogorithm Based on Cosin-window and Interpolation for Harmonic Analysis in Power System," Electrotechnical Journal, vol. 10, 2004, pp.61-64.

[6] Y. M. Zhong, B. P. Tang ang S. R. Qin, "Argument of Some Problems in the Calculation of DFT," Journal of Chongqing University(Natural Science Edition), vol. 24(3), May.2001, pp.1-4.

[7] Y. D. Wang and J. Wang, the Basis of Random Signal Analysis. Beijing: Electronic Industry, 2013.

[8] A. Q. Xu, H. Wei, D. G. Wang and D. Q. Yin, "Research on Time-frequency Method for Estimating SNR of Sine Signal Based on Matlab," Journal of Test nad Measurement Technology, vol. 26, 2012.

[9] S. C. Li, T. C. Ye and J. H. Xu, "Novel Method for Estimating SNR of Sine Signal in Communication System Simulation," Electronic Measurement Technology, vol. 34(3), Mar. 2009, pp.5659.

[10] C. B. Ma, "Application of MATLAB on Smooth Processing of Engineering Test Signal," Technological Development of Enterprise, vol. 32,Jun. 2013, pp. 39-42. 